\title{
Le squelette érythrocytaire et les maladies génétiques de la forme du globule rouge
}

Le squelette érythrocytaire est une assemblée de protéines tapissant la face interne de la membrane plasmique. Il confère aux hématies la souplesse et la résistance qui leur sont nécessaires. Ses protéines, principalement la spectrine, l'actine et la protéine 4.1 , leur assemblage et leur amarrage à des protéines transmembranaires ont été disséqués. L'elliptocytose et la sphérocytose héréditaires constituent par excellence des maladies génétiques du squelette érythrocytaire. Elles résultent d'un extraordinaire éventail de mutations. Ces dernières contribuent à cerner les relations structure-fonction au sein du squelette et parfois permettent de retracer, par bribes, l'histoire des populations concernées.

\section{Jean Delaunay Didier Dhermy}

\section{ADRESSE}

J. Delaunay : professeur des universités. Unité de recherches associée 1171 du Cnrs, génétique moléculaire humaine, faculté de médecine Grange-Blanche, 69373 Lyon Cedex 08 , France.

D. Dhermy : directeur de recherches au Cnrs. Inserm U. 160 (pathologie cellulaire et moléculaire en hématologie), hôpital Beau-

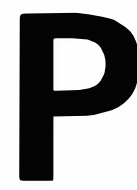

endant les quelque quatre mois de son périple à travers l'organisme, la " carrosserie " du globule rouge doit être souple et robuste à la fois. Souple, afin de négocier le franchissement d'étroits capillaires, et notamment les sinus spléniques; robuste, pour subir les turbulences sanguines régnant dans les gros vaisseaux. C'est une assemblée moléculaire, appelée squelette érythrocytaire, qui confere aux hématies les caractéristiques attendues.

Nous tenterons de dégager les avancées majeures réalisées, au cours de la décennie 1980, à propos du squelette érythrocytaire. Maintenant que sont connues, en tout ou partie, les séquences codantes, l'organisation des gènes, les questions brûlantes sont devenues la structure tridimensionnelle des protéines ainsi que les modalités de l'expression de leurs gènes. Spectrine, ankyrine, protéine $4.1 \ldots$, loin d'être cantonnées au globule rouge, sont quasi ubiquitaires, apparaissant ici et là sous des isoformes spécifiques et contribuant de la sorte à plusieurs aspects de la différenciation cellulaire. Tout ce que l'on peut apprendre sur le squelette érythrocytaire est donc à même de faciliter l'accès aux systèmes homologues, mais plus complexes, tels qu'ils existent dans d'autres types cellulaires.

Nous placerons aussi l'accent sur les bases moléculaires des maladies de la forme du globule rouge, telles que l'elliptocytose et la sphérocytose héréditaires. On a relié ce caractère com- 


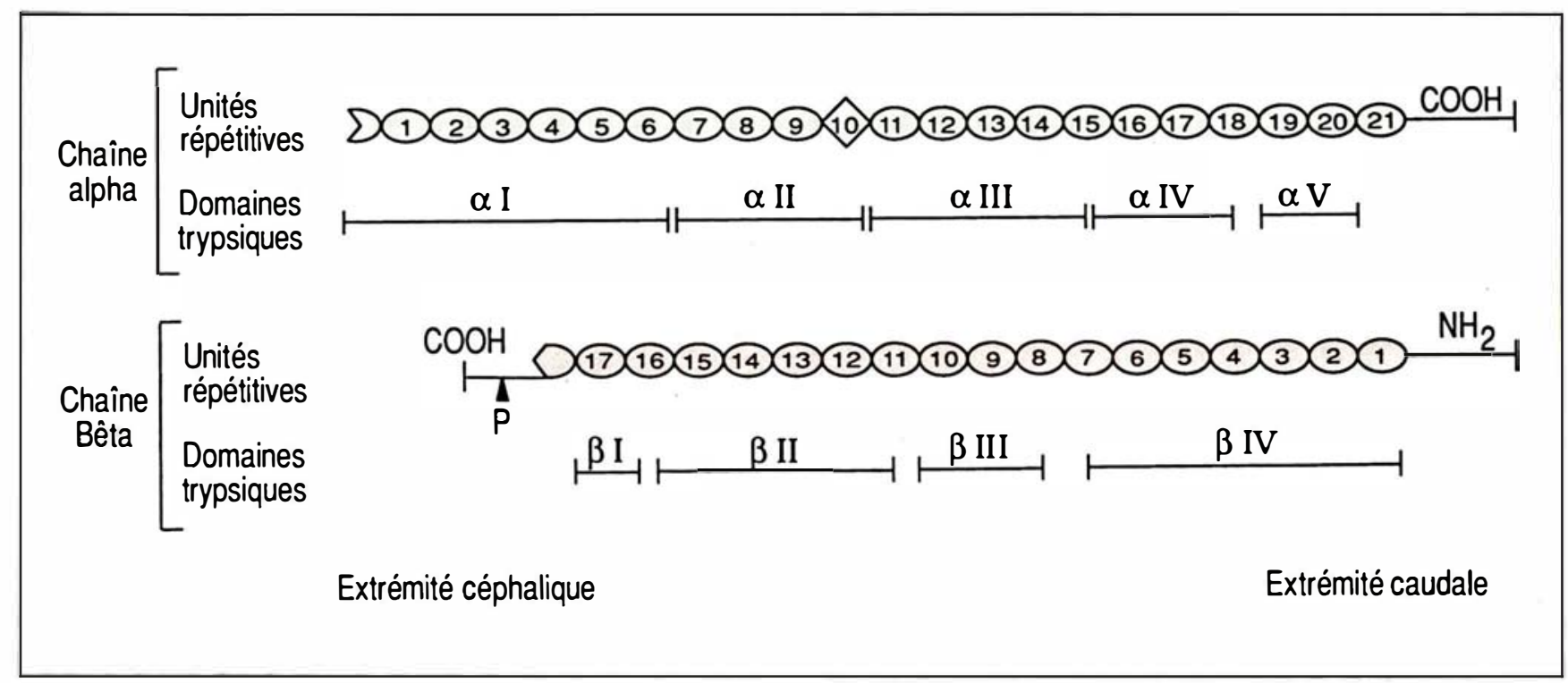

Figure 1. Modèle du dimère de la spectrine. Les unités répétitives de 106 acides aminés sont figurées par des symboles ovales. L'unité $\alpha 10$ n'a pas d'homologie avec les unités répétitives. Les extrémités $\mathrm{N}$-terminale de la chaîne $\alpha$ et C-terminale de la chaîne $\beta$ sont complémentaires l'une de l'autre, reconstituant la structure complète d'une unité répétitive. L'extrémité C-terminale (portion ultime) de la chaîne $\beta$ n'a cependant plus d'homologie avec les unités répétitives et possède les sites de phosphorylation symbolisés par la lettre $P$. Les extrémités C-terminale de la chaîne $\alpha$ et $\mathrm{N}$-terminale de la chaîne $\beta$ (soit les extrémités du tétramère de spectrine) présentent de fortes homologies avec les extrémités de l' $\alpha$-actinine (domaine de liaison avec l'actine). La correspondance proposée par D. Speicher [2] entre unités répétitives et domaines trypsiques est également représentée.

plexe, que représente encore la morphologie d'une cellule, à nombre de mutations définies avec la plus haute résolution. Par-delà des phénotypes relativement univoques a éclaté un formidable polymorphisme génotypique, ouvrant des perspectives sur les relations structure-fonction au sein du squelette érythrocytaire tout comme sur l'histoire des populations humaines concernées.

\section{Les protéines du squelette érythrocytaire}

La spectrine, l'actine et la protéine 4.1 sont les trois principales protéines du squelette érythrocytaire, qui est situé contre la face interne de la bicouche lipidique (pour revue détaillée voir [1]).

La spectrine. La spectrine est un hétérodimère fibrillaire $\alpha \beta$ composé d'une chaîne $\alpha$ et d'une chaîne $\beta$ (Tableau 1 ; figure 1 ). On lui reconnaît une extrémité céphalique et une extrémité caudale. Elle peut être découpée, par hydrolyse enzymatique ménagée [2], en . larges domaines $\mathrm{m} / \mathrm{s} n^{\circ} 6$ vol. 6 , juin 90 numérotés $\alpha \mathrm{I}$ à $\alpha \mathrm{V}$, et $\beta \mathrm{I}$ à $\beta \mathrm{IV}$. A ces domaines, issus de points de clivage hypersensibles à la trypsine, correspondent des fonctions distinctes. Insistons sur le domaine $\alpha \mathrm{I}$ $(80 \mathrm{kDa})$ et $\beta \mathrm{I}(28 \mathrm{kDa})$, qui occupent l'extrémité céphalique du dimère et contiennent, respectivement, les positions $N$ - et $C$-terminales des chaînes $\alpha$ et $\beta$ (celles-ci sont donc antiparallèles au sein du dimère). Le domaine $\beta \mathrm{I}$ est phosphorylable ; néanmoins on ignore la signification biologique de sa phosphorylation. L'étude des séquences, menée au niveau des polypeptides, puis des $\mathrm{ADN}_{c}$, mit en évidence une structure hautement récurrente faite de répétitions de 106 acides aminés (respectivement 21 et 17 répétitions sur les chaînes $\alpha$ et $\beta$ ). Chaque répétition se décompose à son tour en trois hélices : 1,2 et 3 . Des singularités interrompent néanmoins cette régularité. Aux extrémités des chaînes se trouvent des répétitions incomplètes, voire des segments de structure différente. La répétition $\alpha 10$ présente des homologies avec le produit de l'oncogène src.
L'actine. L'actine érythrocytaire fait partie des actines $\beta$. Apte à polymériser, elle voit néanmoins sa polymérisation s'arrêter à 15 monomères environ, à cause de la protéine 4.9 , qui est une molécule de " coiffage" de l'actine.

La protéine 4.1. A la protéine 4.1, on reconnaît quatre domaines par hydrolyse ménagée en présence de chymotrypsine. Chaque domaine est désigné par son poids moléculaire (de la position $N$-terminale à la position $C$-terminale) : $30,16,8-10$ et $24 \mathrm{kDa}$.

Autres protéines. D'autres protéines sont associées au squelette érythrocytaire : myosine, tropomyosine, tropomoduline. La tropomoduline inhibe la liaison de la tropomyosine à l'actine. Affirmer que ces protéines sont encore opérantes, dans l'hématie circulante, serait incertain. Peutêtre ne s'agit-il que de reliques pertinentes à des stades antérieurs de l'érythropoïèse. Mentionnons que spectrine (chaîne $\beta$ ), ankyrine (domaine de $67 \mathrm{kDa}$; voir plus loin), protéine 4.1 et 4.9 sont phosphoryla- 


\begin{tabular}{|l|c|c|c|}
\hline \multicolumn{3}{|c|}{ Tableau I } \\
\multicolumn{3}{|c|}{ QUELQUES CARACTÉRISTIQUES DES PROTÉINES DU SQUELETTE } \\
ÉRYTHROCYTAIRE
\end{tabular}

\section{RÉFÉRENCES}

1. Delaunay J, Alloisio N, Morlé L, Pothier B. The red cell skeleton and its genetic disorders. In : Molecular Aspects of Medicine, Baum H, Gergely J, Fanburg BL, eds. Oxford, Pergamon Press 1990; 11 : 161-241

2. Speicher DW, Morrow JS, Knowles WJ, Marchesi VT. A structural model of human erythrocyte spectrin : Alignment of chemical and functional domains. $J$ Biol Chem 1982 ; 257 : 9093-101

3. Lazarides E. From genes to structural morphogenesis : the genesis and epigenesis of a red blood cell. Cell $1987 ; 51$ : 345-56

4. Hanspal M, Palek J. Synthesis and assembly of membrane skeletal proteins in mammalian red cell precursors. J Cell Biol $1987 ; 105: 1417-24$.

5. Sahr KE, Tobe T, Scarpa A, et al Sequence and exon-intron organization of the DNA encoding the $\alpha \mathrm{I}$. domain of human spectrin. J Clin Invest 1989 ; 84 : 1243-52.

6. Winkelmann JC, Leto TL, Watkins PC, et al. Molecular cloning of the cDNA for human erythrocyte $\beta$-spectrin. Blood 1988 ; 72 : 328-34.

7. Lux S, John K, Shaklev O, et al. Red cell ankyrin is located on chromosome 8 and is deleted or defective in some patients with hereditary spherocytosis. Blood 1988 ; 72 (suppl) : 46a.

8. Conboy J, Mohandas N, Tchernia G, Kan YW. Molecular basis of hereditary elliptocytosis due to protein 4.1 deficiency. $N$ Engl J Med 1986 ; 315 : 680-85.

9. Conboy JG, Ghan J, Mohandas N, Kan YW. Multiple protein 4.1 isoforms produced by alternative splicing in human erythroid cells. Proc Natl Acad Sci USA 1988 ; 85 : 9062-65.

10. Inaba $\mathrm{M}$, Macde $\mathrm{Y}$. $O-N$-acetyl-Dglucosamine moiety on discrete peptide of multiple protcin 4.1 isoforms regulated by alternative pathways. J Biol Chem 1989 ; 264: 18149-55

11. Tang TK, Leto TL, Correas I, Alonso MA, Marchesi VT, Benz Jr EJ. Selective expression of an erythroid-specific isoform of protein 4.1. Proc Natl Acad Sci USA 1988 ;

${ }^{a}$ : près des loci Duffy $;^{b}$ : près du groupe de loci Rhésus $;{ }^{c}$ : trimères $;{ }^{d}$ : monomères.

bles en des sites précis, qui en présence de protéine kinases stimulées par l'AMP ${ }_{c}$, qui en présence de protéine kinases C. Là encore, on débat du caractère physiologique des phosphorylations dans les hématies circulantes.

\section{Les articulations entre protéines}

Spectrine, actine et protéine 4.1 s'articulent, à travers des interactions dites horizontales, pour former un réseau aux mailles régulières (figure 2). Par des interactions verticales, elles s'amarrent à des protéines transmembranaires.

Les deux interactions horizontales. Deux dimères de la spectrine s'associent, tête à tête, pour former un tétramère, forme prédominant in situ. Rappelons que cette interaction met en jeu les régions $N$-terminale (domaine $\alpha \mathrm{I}$ ) de la chaîne $\alpha$.

Par son extrémité caudale (domaine $\alpha \mathrm{V}$ et $\beta \mathrm{IV}$ ), le dimère de la spectrine se fixe à l'actine - deuxième interaction horizontale, que "verrouille" la protéine 4.1. L'ensemble forme le complexe ternaire. L'adducine, protéine liant la calmoduline, resserrerait également la liaison actine-spectrine. A noter que la région caudale de la spectrine a des homologies structurales avec l' $\alpha$-actinine et (chaîne $\beta$ ) avec la région $N$-terminale de la dystrophine (la structure récurrente globale de la spectrine constituant une autre ressemblance avec la dystrophine).
Les deux interactions verticales. A $20 \mathrm{~nm}$ de son site d'autoassociation, la spectrine (domaine $\beta$ II) interagit avec la portion cytoplasmique (43 kDa) de la bande 3. Par sa portion transmembranaire, la bande 3 , glycoprotéine dont il existe $10^{6}$ copies par hématie, catalyse la diffusion des anions $\mathrm{C}^{-}$et $\mathrm{HCO}_{3}{ }^{-}$. Pour autant, le contact spectrinefragment $43 \mathrm{kDa}$ n'est pas direct, mais se fait par l'entremise d'une tierce protéine appelée ankyrine. Celle-ci a trois domaines, définis à nouveau par leurs poids moléculaire $(N \rightarrow C): 89,67$ et $55,5 \mathrm{kDa}$. Le premier interagit avec la bande 3, le second avec la spectrine. Une autre protéine encore intervient, nommée protéine 4.2, qui module(rait) l'interaction ankyrine-bande 3 . A noter que la protéine 4.2 comporte des homologies de séquence avec des transglutaminases sans néanmoins présenter cette activité enzymatique.

L'autre interaction verticale met en jeu la protéine 4.1 (domaine $30 \mathrm{kDa}$ ) et (i) le fragment $43 \mathrm{kDa}$ de la bande 3 et (ii) la portion cytoplasmique de sialoglycoprotéines (glycophonines), protéines lourdement sialylées dans leur partie extracellulaire et portant différents antigènes des groupes sanguins.

Précisons ici que la séquence d'assemblage du squelette érythrocytaire, et de son amarrage à la membrane, fut disséquée par le menu au cours de l'érythropoiièse chez le poulet [3], puis chez les mammiferes [4]. Un trait saillant est la synthèse d'un 
immense excès de spectrine, qui est aussitôt dégradé. Les raisons de cet apparent gaspillage sont inconnues.

\section{Les gènes des protéines du squelette membranaire}

La connaissance des gènes et de leurs transcrits codant pour les principales protéines du squelette membranaire érythrocytaire a été déterminante dans de nombreux domaines :

- elle seule a bien souvent permis d'accéder à la connaissance de la séquence primaire de la protéine; - elle a également été déterminante dans la compréhension de la pathologie génétique de ces protéines; - enfin, elle devrait permettre de mieux caractériser et de comprendre les mécanismes qui sont à l'origine des formes érythroïdes et non érythroïdes de ces protéines (familles multigéniques ; épissages alternatifs) et d'analyser les différences qui existent pour un même système cellulaire entre différentes espèces.

Le gène de la chaîne $\alpha$ de la spectrine érythrö̈de. Le premier clone contenant un fragment du gène $\alpha$ de la spectrine érythroïde a été isolé par L. Cioe et P. Curtis en 1985 à partir d'une banque érythroïde murine. Ce clone a permis ensuite d'isoler un clone $\mathrm{ADN}_{\mathrm{c}}$ humain codant pour les répétitions 14 à 17 de la chaîne $\alpha$ de la spectrine. La taille du messager de la chaîne $\alpha$ de la spectrine déterminée par Northern blot est de $8 \mathrm{~kb}$. La localisation chromosomique du gène de la chaîne $\alpha$, ainsi que d'autres gènes, est donnée dans le Tableau I. La grande taille de l'ARNm de la chaîne $\alpha$ rendait bien sûr difficile

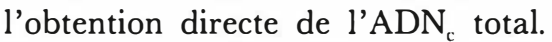
Des clones en 5' et 3' ont été isolés à partir de banques de foie fotal et de moelle osseuse humaine, permettant ensuite par criblages successifs une "marche" sur l'ADN let $_{\text {c }}$ " l'obtention de sa séquence complète (B. Forget, communication personnelle). L'organisation exon-intron de la partie du gène spectrine codant pour le domaine $\alpha 1$ est maintenant connue. Cette partie comprend 12 exons dont les tailles varient de 43 à 240 paires de bases ( $\mathrm{pb}$ ), celles des introns allant de 0,1 à $2 \mathrm{kpb}$ [5]. Il n'existe pas de rapport évident entre l'organisation exon-intron du gène et la structure répétitive de 106 acides aminés.

Le gène de la chaîne $\beta$ de la spectrine érythroïde. Des $\mathrm{ADN}_{c}$ ont été isolés d'une banque érythroïde humaine. Ils codent pour approximativement la moitié $\mathrm{COOH}$ terminale de la chaîne $\beta$ de la spectrine [6]. On retrouve une structure en unités répétitives identique à celle décrite pour la chaîne $\alpha$. L'ADN ${ }_{c}$ complet de la chaîne $\beta$ a été récemment caractérisé. Il code pour une protéine de 2317 acides aminés $(246 \mathrm{kDa})$.

Le gène de l'ankyrine érythroïde. Un premier clone d'ADN ${ }_{c}$ a été isolé d'une banque d'expression de réticulocytes humains. Ce clone code pour une partie du domaine $\mathrm{COOH}$ terminal de l'ankyrine [7]. Un autre clone d'ADN c a été aussi isolé d'une banque de réticulocytes humains par S. Lambert et al. Ce clone est situé plus en 5' du précédent et contient des structures répétitives codant pour des motifs de 33 acides aminés. Un Southern blot d'ADN génomique humain hybridé avec cette sonde indique la présence

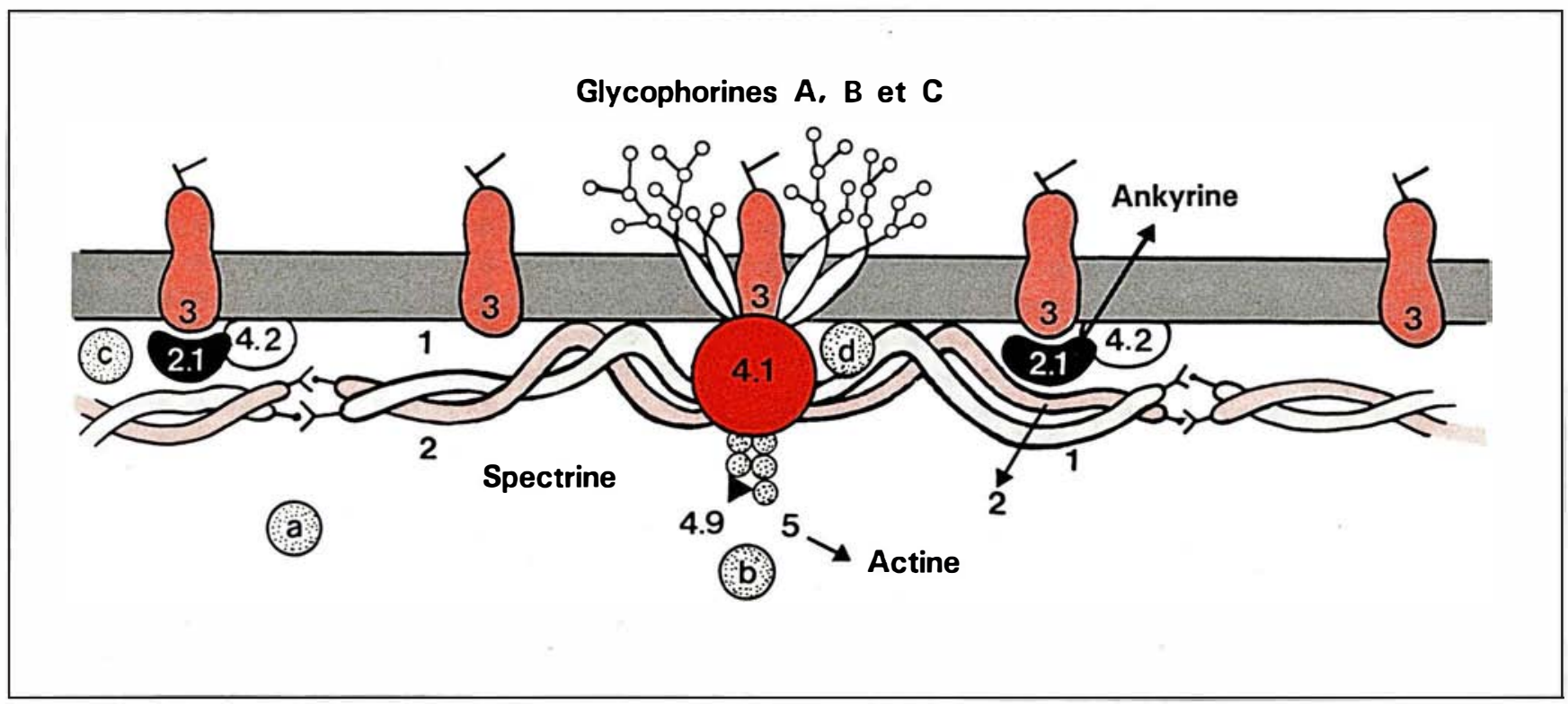

Figure 2. Coupe transversale du squelette érythrocytaire. Le squelette érythrocytaire se situe juste au-dessous de la membrane érythrocytaire que traversent, à intervalles rapprochés, la bande 3 (transporteur des anions) et, de façon plus éparse, les glycophorines $A, B$ et $C$. 1 : chaîne $\alpha$ de la spectrine; 2 : chaîne $\beta$ de la spectrine; 2.1 : ankyrine; 4.1 : protéine 4.1 ; 4.2 : protéine 4.2. ; 4.9 : protéine de coiffage de l'actine ; 5 : actine. a : site d'autoassociation du dimère de la spectrine. b : complexe ternaire spectrine - actine - protéine 4.1. c : articulation spectrine - ankyrine - protéine 4.2 - bande 3. $d$ : interaction de la protéine 4.1 avec la bande 3 et les glycophorines. 


\section{RÉFÉRENCES}

12. Lux SE, John KM, Kopito RR, Lodish $\mathrm{HF}$. Cloning and characterization of band 3 , the human erythrocyte anion-exchange protein. Proc Natl Acad Sci USA 1989 ; 86 : 9089-93.

13. Garbarz M, Lecomte MC, Feo C, et al. Hereditary pyropoikilocytosis and elliptocytosis in a white french family with the Spectrin $\alpha^{1 / 74}$ variant related to a CGT To CAT codon change (Arg To His) at position 22 of The Spectrin $\alpha^{\mathrm{I}}$ domain. Blood 1990 ; 75 : 1691-8.

14. Morlé L, Roux AF, Alloisio N, et al. Two elliptocytogenic $\alpha^{1 / 74}$ variants of the spectrin $\alpha \mathrm{I}$ domain. Spectrin Culoz and spectrin Lyon, mutated at position 40 (GGT $\rightarrow$ GTT; Gly $\rightarrow \mathrm{Val}$ ) and at position 43 (CTT $\rightarrow$ TTT ; Leu $\rightarrow$ Phe), respectively. $J$ Clin Invest. In press.

15. Tse WT, Costa FF, Lecomte MC, et al. An Ala to Pro substitution in the $\beta$ spectrin chain causes $\alpha^{1 / 74}$ hereditary elliptocytosis (HE). Blood 1989: 74 (suppl 1), 105a (Abstr).

16. Dhermy D, Lecomte MC, Garbarz M, et al. Spectrin $\beta$-chain variant associated with hereditary elliptocytosis. J Clin Invest $1982 ; 70: 707-15$.

17. Pothier B, Morlé L, Alloisio N, et al. Spectrin Nice $\left(\beta^{220-216}\right)$ : a shortened $\beta$ chain variant associated with an increase of the $\alpha^{1 / 74}$ fragment in a case of elliptocytosis. Blood 1987 ; 69 : 1769-65.

18. Conboy J, Kim R, Agre P, Tchernia G, Kan YW, Mohandas N. Novel alterations of erythroid protein 4.1 gene expression in patients with hereditary elliptocytosis. Blood 1989 : 74 (suppl 1), 181a (Abstr).

19. Féo C, Fischer S, Piau JP, Grange MG, Tchernia G. Première observation de l'absence d'une protéine de la membrane érythrocytaire (bande 4.1) dans un cas d'anémie elliptocytaire familiale. Nouv Rev Fr Hématol $1980 ; 22$ : 315-25.

20. Ghanem A, Pothier B, Maréchal J, et al. A haemolytic syndrome associated with the complete absence of red cell membrane protein 4.2 in two Tunisian siblings. Brit $J$ Haematol (sous presse)

21. Ata K, Kanzaki A, Yawata Y. Deficiency of membrane band 4.2 in red cells of two cases of congenital hemolytic anemia (HS and HE). J Cell Biochem 1989: 13B (suppl), 228 (Abstr CD400).

22. Alloisio N, Morlé L, Dorléac E, et al. The heterozygous form of 4.1 ( - ) hereditary elliptocytosis (the 4.1 ( - ) trait). Blood d'un seul gène codant pour l'ankyrine.

Gène de la protéine 4.1. Des clones d'ADN ${ }_{c}$ recouvrant toute la partie codante du gène de la protéine 4.1 ont été isolés d'une banque d'expression de réticulocytes humains. Un Northern blot hybridé avec cette sonde indique un ARNm de 5,6 kb [8]. L'étude d'autres clones isolés de la banque érythroïde a révélé une hétérogénéité dans la structure de la protéine 4.1. Il existe en effet différentes isoformes de cette protéine. Les séquences de ces isoformes diffèrent soit au niveau du domaine de fixation de la spectrine sur la protéine 4.1 (domaine de 8-10 kDa), soit au niveau du domaine $\mathrm{COOH}$ terminal (domaine de $24 \mathrm{kDa}$ ), soit encore au sein du domaine $\mathrm{NH}_{2}$ terminal (domaine de $30 \mathrm{kDa}$ ) [9]. Plus précisément, les séquences d'ADN codant pour ces isoformes ont de très longues régions de parfaite homologie interrompues par l'insertion ou la délétion de séquences nucléotidiques correspondant à celles d'exons du gène de la protéine 4.1. Ainsi, deux isoformes du domaine de $8-10 \mathrm{kDa}$ diffèrent par la délétion d'une séquence codant pour 21 acides aminés dans l'un d'entre eux; dans le domaine $\mathrm{COOH}$ terminal, trois isoformes diffèrent par l'expression alternative d'un peptide de 43 ou de 34 acides aminés ou des deux. L'analyse des séquences génomiques codant pour le peptide de 34 acides aminés montre que la séquence codante est flanquée par des séquences introniques, correspondant à des sites accepteur et donneur d'épissage, suggérant que l'addition ou la délétion de cette séquence est le résultat d'un épissage alternatif. En outre, ce peptide de 34 acides aminés est glycosylé par un unique résidu $O-N$ acétyl-D-glucosamine [10]. Ainsi les cellules érythroïdes, par des mécanismes complexes, expriment différentes isoformes de la protéine 4.1 qui pourraient être impliquées aux différents stades de la différenciation érythroïde.

Des clones d'ADNc codant pour des isoformes de la protéine 4.1 ont été aussi isolés d'une banque de cellules lymphoïdes MOLT4 par criblage avec une sonde pour la protéine 4.1 érythroïde [11]. La séquence de ces clones a montré l'absence du motif nucléotidique codant pour la séquence de 21 acides aminés du domaine $8 \mathrm{kDa}$. Cette séquence est spécifique de la protéine 4.1 érythroïde, car elle est spécifiquement exprimée dans les cellules de Friend induites.

Gène de la protéine bande 3. Après celle de la bande 3 de l'érythrocyte de poulet, la séquence protéique complète de la bande 3 de l'érythrocyte de souris, puis celle de la bande 3 humaine [12] ont été déduites des séquences nucléotidiques de l'ADNc. Les séquences correspondant aux domaines cytoplasmiques different, surtout au niveau de l'extrémité $\mathrm{N}$-terminale (56 premiers acides aminés). En revanche, les domaines membranaires (segments transmembranaires et boucles externes) sont hautement conservés. Le gène de la bande 3 érythrocytaire chez l'homme a été localisé sur le chromosome 17 et un seul transcrit de $4,7 \mathrm{~kb}$ est présent dans les réticulocytes.

\section{L'elliptocytose héréditaire}

L'elliptocytose héréditaire désigne une catégorie d'anémies hémolytiques signalées par des hématies elliptiques (figure 3). Il était logique que les mutations responsables fussent cherchées dans le squelette érythrocytaire. Variables dans leurs manifestations cliniques, dans leur mode de transmission (en général complètement ou partiellement dominantes, elles sont parfois récessives), les elliptocytoses héréditaires le sont bien davantage encore par le polymorphisme des lésions génomiques sous-jacentes. Audelà de son aspect descriptif, l'identification de mutations naturelles permet des déductions sur la fonction des différents domaines affectés. Nous présenterons quelques exemples choisis.

L'elliptocytose héréditaire $\mathrm{Sp} \alpha^{\mathrm{I} / 74}$. Que signifie, d'abord, cette appellation rocailleuse ? Qu'une déviation de l'hydrolyse ménagée de la spectrine, en présence de trypsine, se fait jour. Au lieu d'apparaître de façon majoritaire sous la forme d'un fragment de $80 \mathrm{kDa}$, le domaine $\alpha \mathrm{I}$ donne avant tout, désormais, un fragment de $74 \mathrm{kDa}$, reflétant un cli- 


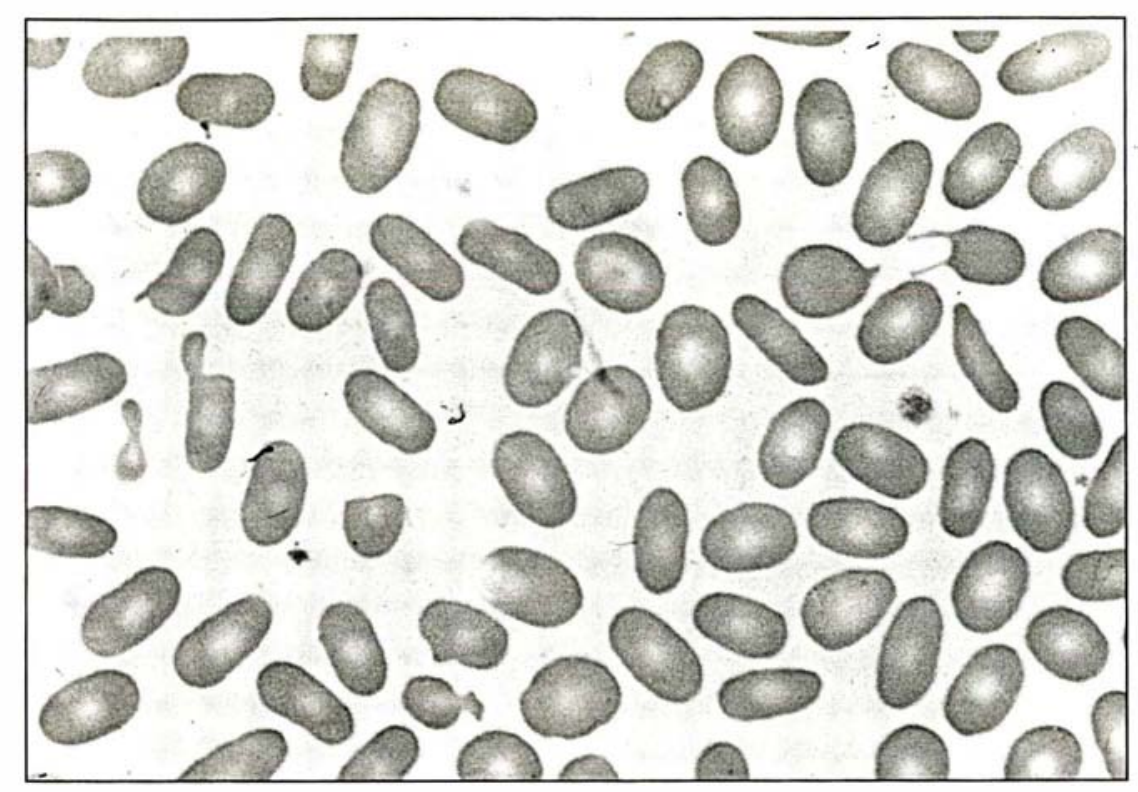

Figure 3. Frottis d'elliptocytose. Tous les globules rouges sont des elliptocytes, avec un degré d'ellipticité variable. A l'elliptocytose s'ajoute une tendance au bourgeonnement, témoin de la fragilité mécanique des cellules. La lésion moléculaire sous-jacente à ce cas particulier d'elliptocytose est une mutation ponctuelle de la chaîne $\alpha$ de la spectrine ( $\alpha$ l Leu $\rightarrow$ Phe; CTT $\rightarrow$ TTT).

vage accru après les résidus Arg 39 et/ou Lys 42. De cette anomalie de comportement de substrat, on infere l'existence d'une mutation alentour. C'est une série de mutations qui fut trouvée en effet. Certaines, comme cela paraît logique, se situent dans la région $N$-terminale de la chaîne $\alpha$, à proximité du point de coupure devenu hypersensible, ainsi que nous l'avons montré en collaboration avec les groupes de B.G. Forget et de J. Godet $: \alpha$ I 22 Arg $\rightarrow$ His $($ CGT $\rightarrow$ CAT) [13] ; $\alpha$ I 40 Gly $\rightarrow$ Val (GGT $\rightarrow$ GTT) ; $\alpha$ I 43 Leu $\rightarrow$ Phe (CTT $\rightarrow$ TTT) [14].

Il $\mathrm{y}$ a donc là un segment polypeptidique sur lequel les mutations présentent un haut risque elliptocytogène. Contre toute attente, d'autres mutations responsables de l'anomalie $\mathrm{Sp} \alpha^{1 / 74}$ apparurent dans la région Cterminale, située en face, de la chaîne $\alpha$. Nous reviendrons plus loin sur le type de mutations éclos à cet endroit, car beaucoup sont de surcroît d'un type particulier (délétion d'un segment polypeptidique).

Ce qui doit être souligné pour le moment, c'est que les mutations $\alpha^{1 / 74}$ se rencontrent sur une hélice 3 isolée, précédant la première répéti$\mathrm{m} / \mathrm{s} n^{\circ} 6$ vol. 6 , juin 90 et pouvaient dans deux cas apparâ̂tre comme le résultat d'altérations $d e$ novo. Des raccourcissements comparables furent rapportés pour la chaîne $\alpha$ et la protéine 4.1. Il s'agit donc, en définitive, d'un cas de figure banal. Pourquoi ?

En ce qui concerne la région $C$ terminale de la chaîne $\beta$, les premières indications montrent l'existence, dans certains cas, de mutations à des jonctions exon-intron, suivies de perte d'exons excisés comme s'ils appartenaient aux introns et, le cas échéant, de décalage de la phase de lecture, puis de codon sto $p$ prématuré. On peut anticiper que, dans cette région, les phénomènes d'épissage sont plus complexes, les régions " consensus" directrices d'épissage plus rapprochées, donc les ratées de l'épissage plus fréquentes. Ainsi en va-t-il également de la protéine 4.1 qui doit, nous l'avons signalé, tirer toutes ses isoformes d'un unique gène 4.1 dont le transcrit est épissé selon plusieurs schémas.

Des variants allongés de protéines du squelette érythrocytaire ont aussi été décrits, mais plus rarement. Par exemple, un mutant elliptocytogène de la protéine 4.1 de $95 \mathrm{kDa}$ (au lieu de $80 \mathrm{kDa}$ normalement) résulte de la duplication du segment $\mathrm{Lys}_{407} \rightarrow \mathrm{Gln}_{529}$ : cette duplication résulte à son tour d'une insertion étendue dans le gène protéine 4.1 [18].

Les anomalies de type thalassémique. L'absence totale d'une protéine entraîne en règle un tableau clinique notable ou grave. Pour des allèles pathologiques peu fréquents, une homozygotie a quelque probabilité de se produire chez les descendants de couples consanguins, chaque membre étant porteur du trait. Ainsi Féo et al. [19] découvrirent-ils une absence totale de la protéine 4.1 chez trois enfants issus d'un mariage consanguin, en Grande Kabylie (figure 5). On sait aujourd'hui que la lésion moléculaire responsable est la perte d'un fragment d'ADN comportant, entre autres, l'exon qui inclut le codon d'initiation de la protéine 4.1 (érythrocytaire) [18]. Des études d'haplotypes laissent prévoir que les allèles $4.1(-)$ sont dus, là encore, à tout un spectre de mutations, mais faute de réarrangement génique manifeste dans des familles différen- 


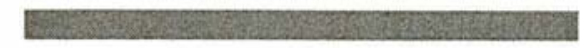

\section{RÉFÉRENCES}

23. Feddal S, Brunet G, Roda L, et al. Molecular analysis of a variety of $4.1(-)$ hereditary elliptocytosis found in the French province of Savoy (manuscrit soumis).

24. Lecomte MC, Dhermy D, Gautero H, Bournier O, Galand C, Boivin P. L'elliptocytose héréditaire en Afrique de l'Ouest : fréquence et répartition des variants de la spectrine. CR Acad Sci Paris 1988; 306 : 43-6.

25. Roux AF, Morlé F Guetarni D, et al. Molecular basis of $\mathrm{Sp} \alpha^{1 / 65}$ hereditary elliptocytosis in North Africa : insertion of a TTG triplet between codons 147 and 149 in the $\alpha$-spectrin gene from five unrelated families. Blood 1989 ; 73 : 2196-201.

26. Sahr KE, Garbarz M, Dhermy D, et al. Use of the polymerase chain reaction for the detection and characterization of mutations causing hereditary elliptocytosis. In : Cohen CM, Palek J, eds. Cellular and Molecular Biology of Normal and Abnormal Erythroid Membranes, UCLA Symposia on Molecular and Cellular Biology, New Series, vol. 118, New York: Alan R Liss Inc (sous presse).

27. Goodman SR, Shiffer KA, Casoria LA, Eyster ME. Identification of the molecular defect in the erythrocyte membrane skeleton of some kindreds with hereditary spherocytosis. Blood $1982 ; 60$ : 772-84.

28. Wolfe LC, John KM, Falcone JC, Byrne AM, Lux SE. A genetic defect in the binding of protein 4.1 to spectrin in a kindred with hereditary spherocytosis. $N$ Engl $J$ Med $1982 ; 60$ : 772-84.

29. Agre P, Orringer EP, Bennett V. Deficient red-cell spectrin in severe, recessively inherited spherocytosis. $N$ Engl J Med 1982 ; $306: 1155-60$

30. Agre P, Casella JF, Zinkham WH, McMillan C, Bennett V. Partial deficiency of erythrocyte spectrin hereditary spherocytosis. Nature $1985 ; 314: 380-3$.

31. Kimberling WJ, Taylor RA, Chapman RG, Lubs HA. Linkage and gene localization of hereditary spherocytosis (HS). Blood 1978 ; 52 : 859-67.

32. Davies KA, Lux SE. Hereditary disorders of the red cell membrane skeleton. Trends in Genetics $1989 ; 5$ : 222-7.

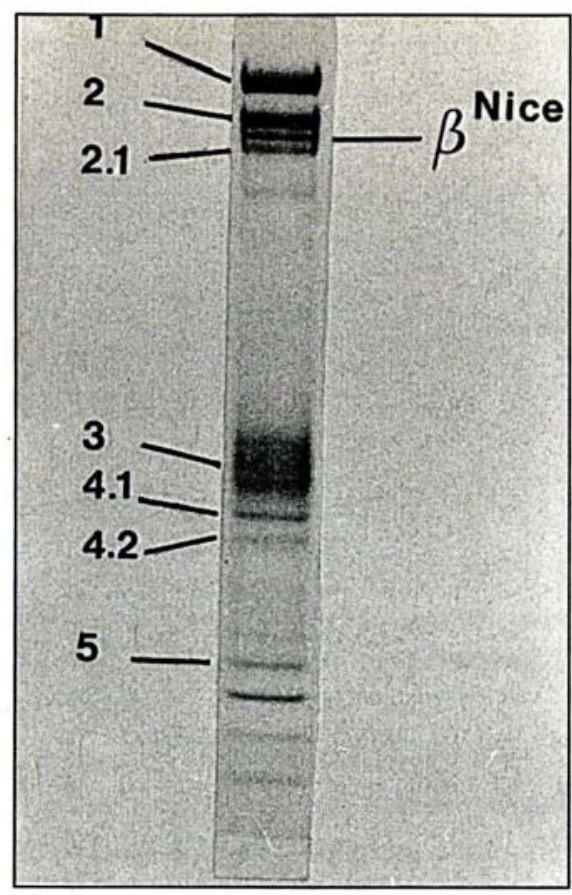

Figure 4. Un exemple de chaîne polypeptidique tronquée [17]. La chaîne $\beta$ de la spectrine apparaît sous la forme de la bande 2 normale $(220 \mathrm{kDa})$ et d'une chaîne $\beta$ raccourcie ( $\left.\beta^{\text {Nice }} ; 216 \mathrm{kDa}\right)$. L'amputation porte sur une trentaine d'acides aminés de la région $\mathrm{N}$-terminale et emporte les sites de phosphorylation. Des variations notables de la taille des chaînes polypeptidiques sont fréquemment rencontrées dans les protéines du squelette érythrocytaire et sont presque toujours responsables d'altérations morphologiques.

tes, aucune autre mutation n'a encore été élucidée de façon fine.

Bien que le tableau clinique réalisé ne corresponde ni à une elliptocytose ni à une sphérocytose, mais semblet-il à un syndrome original, nous citerons ici l'absence totale de protéine 4.2, découverte chez deux scurs issues d'un mariage consanguin, dans l'oasis de Tozeur (figure 5) [20]. Cette anomalie est connue depuis plus de dix ans au Japon (voir plus loin). Il est fort probable que les mutations tunisienne et japonaise soient différentes. D'où, là aussi, un polymorphisme attendu derrière le même phénotype protệique.

Épidémiologie de l'elliptocytose. Nous avons souligné jusqu'à présent le polymorphisme des lésions moléculaires responsables d'elliptocytose. Les lésions elliptocytogènes témoignent donc, comme beaucoup d'autres altérations, du bruit de fond mutationnel que subit en permanence le génome humain. Les changements étant, au pire, de gravité intermédiaire à l'état hétérozygote et inversement ne conférant pas d'avantage évident, elles n'ont tendance ni à disparaître ni à se propager au fil des générations. Mais en est-il toujours ainsi ?

Il existe des isolats dilués, tel le Japon pour l'anémie hémolytique $4.2(-)$ [21], ou encore la vallée du Fier (Haute-Savoie) pour l'elliptocytose héréditaire $4.1(-)$ [22, 23]. Cet enrichissement relatif d'une population, en tel allèle $4.2(-)$ ou 4.1 ( - ), résulte de l'isolement géographique, favorisant la consanguinité et, le cas échéant, de facteurs démogénétiques (grandes fratries).

En Afrique Noire, l'elliptocytose atteint une fréquence endémique (> $1 \%$ ) [24]. En outre, une mutation revient sans cesse : la duplication du codon 148 (elliptocytose $\left.\alpha^{1 / 65}\right)($ TTG ; Leu) de la chaîne $\alpha$ de la spectrine [25, 26]. Pareille mutation, cliniquement asymptomatique à l'état hétérozygote, procure, pense-t-on, un avantage constitutionnel contre la malaria - comme le fait, dans les mêmes populations du reste, l'hémoglobine $\mathrm{S}$.

Une note ethnologique enfin. L'Afrique du Nord, carrefour des civilisations, porte, dans le répertoire génétique des populations d'aujourd'hui, la trace des invasions qui jalonnèrent son histoire. Ne sait-on pas depuis longtemps que cohabitent des hémoglobines venues du sud (hémoglobine $S$ ) et d'autres venues du nord ou du Moyen-Orient (hémoglobine Lepore)? Il en va strictement de même des mutations elliptocytogènes. On y rencontre, originaire du sud, l'elliptocytose $\alpha^{\mathrm{I} / 65}$ vue précedemment et, arrivée du nord ou du MoyenOrient, l'elliptocytose $4.1(-)$.

\section{Sphérocytose héréditaire}

Décrite pour la première fois en 1871, par deux médecins belges C. F. Vanclair et J. B. Masius, puis caractérisée par O. Minkowski et M.A. Chauffard en 1907, la sphérocytose héréditaire est l'une des ané- 


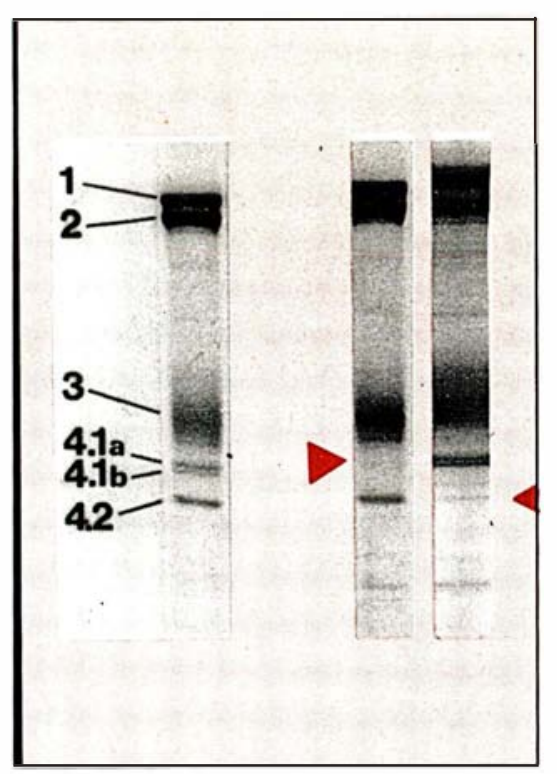

Figure 5. Deux exemples de mutations thalassémiques à l'état hétérozygote. L'absence totale de protéine 4.1, génératrice d'une elliptocytose grave, fut découverte en Kabylie chez trois enfants nés d'un mariage consanguin. L'absence totale de protéine 4.2, qui produit une anémie hémolytique sévère, ne répond cependant ni aux critères de l'elliptocytose ni à ceux de la sphérocytose. Elle fut reconnue à Tozeur, chez deux personnes nées également d'un mariage consanguin. A gauche : fraction du profil électrophorétique normal des protéines de l'ensemble: membrane + squelette érythrocytaires; dans les conditions utilisées, l'ankyrine (bande 2.1) ne se voit pas car elle migre derrière la chaîne $\beta$ de la spectrine (bande 2).

mies hémolytiques héréditaires les plus fréquentes dans les populations d'ethnie blanche (1 pour 6000 environ).

La transmission génétique est habituellement autosomique dominante, mais il faut savoir que, dans 25 à $35 \%$ des cas, aucune anomalie hématologique caractéristique de la maladie n'est retrouvée chez les deux parents. En l'absence le plus souvent d'un support moléculaire connu, il est difficile d'expliquer cette transmission " non dominante ". Trois hypothèses sont avancées : une pénétrance variable, une mutation nouvelle ou une forme réellement récessive sem$\mathrm{m} / \mathrm{s} n^{\circ} 6$ vol. 6 , juin 90 blable à celle décrite dans certaines lignées de souris.

La lésion membranaire qui caractérise le sphérocyte est une diminution de la surface de la membrane par rapport au volume de la cellule. Le sphérocyte est donc une cellule osmotiquement fragile et l'augmentation de cette fragilité reste le meilleur test diagnostique en routine hospitalière.

Étiologie de la sphérocytose héréditaire. En dehors de quelques cas isolés, la ou les anomalies membranaires directement responsables de la maladie restent à découvrir, il existe des modèles murins avec transmission récessive de la sphérocytose héréditaire susceptibles d'aider à la compréhension de la maladie humaine. Dans certaines lignées de souris atteintes d'hémolyse avec sphérocytose existe un déficit quantitatif plus ou moins sévère en spectrine. Les études de synthèse protéique dans les réticulocytes ont mis en évidence la possibilité d'anomalies de synthèse de la chaîne $\alpha$ ou $\beta$ de la spectrine ou de l'ankyrine.

Sphérocytose héréditaire et anomalie de la chaîne $\beta$ de la spectrine. Deux équipes américaines [27, 28] ont découvert, dans trois familles, un défaut d'interaction entre la spectrine et la protéine 4.1 : $40 \%$ des molécules de spectrine du patient étaient incapables d'interagir avec la protéine 4.1 normale. Cette anomalie apparaît liée à un changement conformationnel de l'extrémité $\mathrm{NH}_{2}$ de la chaîne $\beta$ de la spectrine, dont la base génétique n'est pas encore connue mais qui est responsable de l'oxydation d'un résidu de cystéine anormalement exposé. Si cette anomalie de la spectrine apparaît assez bien caractérisée, elle demeure certainement une cause rare de sphérocytose.

Sphérocytose héréditaire et déficit en spectrine. En 1982, P. Agre et al. rapportaient l'étude d'une famille originaire de Caroline du Nord dont la généalogie révélait l'existence de plusieurs mariages consanguins [29]. Chez deux enfants porteurs d'une hémolyse néonatale sévère avec sphérocytose très marquée, l'étude électrophorétique des protéines membranaires révélait un déficit en spectrine de plus de $70 \%$, confirmé par dosage radio-immunologique. Les études cytologiques et biochimiques effectuées chez les deux parents, cousins germains, étaient toutes normales.

A la suite de l'observation de cette famille, P. Agre et al. poursuivirent l'étude quantitative de la spectrine dans les sphérocytoses héréditaires. En 1985 [30], ils rapportèrent l'étude de 14 patients sphérocytaires, tous porteurs d'un déficit en spectrine, estimé par radio-immunodosage entre 15 et $70 \%$, l'intensité de ce déficit apparaissant parallèle à la sévérité de la maladie. En 1986, leur étude était étendue à 33 cas, permettant de préciser que le déficit partiel en spectrine était surtout marqué dans les sphérocytoses héréditaires " non dominantes".

Parmi 6 familles de sphérocytoses héréditaires non dominantes avec déficit en spectrine décrites en 1986 par P. Agre, J. Winkelmann et S. Marchesi mettaient en évidence dans 4 familles, en plus du défaut quantitatif, un défaut qualitatif de la spectrine révélé par l'analyse bidimensionnelle des digestats trypsiques. Cette anomalie était caractérisée par un glissement vers l'acidité de deux peptides caractéristiques du domaine $\alpha$ II. Cette anomalie se trouvait plus ou moins marquée, évoquant un état hétérozygote ou homozygote. Cette étude a été étendue par S. Marchesi et al. à 14 familles de sphérocytoses non dominantes. L'anomalie a été retrouvée dans 9 familles chez des patients sphérocytaires mais également à l'état hétérozygote chez des sujets non affectés. La relation entre ce variant $\alpha \mathrm{II}$, dû à une mutation Ala $\rightarrow$ Asp en position 970 , et la sphérocytose héréditaire n'est pas encore très claire. La manière par laquelle des mutations différentes, affectant la spectrine, produisent tantôt une sphérocytose (cas présent), tantôt une elliptocytose (voir plus haut), n'est pas comprise à ce jour, mais se conçoit du fait de la spécialisation fonctionnelle des différents domaines de la protéine.

Le déficit en spectrine dans la sphérocytose héréditaire peut également être secondaire à un déficit en ankyrine. T. Coetzer et al., dans le laboratoire de J. Palek, ont récemment 
décrit, dans deux cas de sphérocytose (de transmission dominante dans un cas et "non dominante" dans l'autre), un déficit de $50 \%$ de spectrine secondaire à un déficit en ankyrine de même degré. Ce déficit membranaire en ankyrine est probablement dû à une instabilité de la protéine.

On comprendra, à la lecture de ce chapitre étiologique, que le phénotype sphérocytose recouvre plusieurs types d'anomalies quantitatives ou qualitatives, parfois associées, affectant des protéines différentes, et que, dans bien des cas, le déficit primaire reste incertain.

La sphérocytose héréditaire (tout comme l'elliptocytose héréditaire, d'ailleurs) représente un bon exemple pour une approche méthodologique du type repérage de gène candidat. Les données biochimiques réunies chez l'homme, qui recoupent en partie celles recueillies chez la souris, semblent actuellement définir deux possibles chapitres étiologiques : un chapitre spectrine et un chapitre ankyrine. Le gène de la chaîne $\beta$ de la spectrine a été localisé au niveau du bras long du chromosome 14. Des études anciennes avaient déjà permis à W. Kimberling et al. [31] de reconnaître dans certaines familles un lien entre la sphérocytose et le locus des chaînes lourdes d'immunoglobulines, localisé depuis sur le chromosome 14 (14q32.3). Le gène de l'ankyrine chez l'homme a été localisé sur le bras court du chromosome 8. Dans quatre familles antérieurement rapportées dans la littérature et associant sphérocytose et translocation ou délétion du chromosome 8 [32], le gène " sphérocytose " avait déjà pu être fortement associé au segment 8p11.22-8p21.1.

En conclusion, nombreuses sont les lignes de recherche qui se dessinent aujourd'hui, en matière de squelette érythrocytaire, sur les acquis de la décennie écoulée. L'organisation supramoléculaire, jusqu'à l'échelle même de la cellule, se déduira des propriétés individuelles des molécules. Au cours de l'érythropoïèse, la mise en place des pièces protéiques selon un calendrier précis offre un exemple accessible d'édification d'une structure complexe. La régulation de

types de gènes éclaire une facette de la différenciation érythroïde, mais aussi de la différenciation de types cellulaires fort éloignés du globule rouge : neurones, cellules musculaires... La définition au niveau le plus fin des mutations altérant la forme érythrocytaire, outre qu'elle autorise une classification nosologique nouvelle et limpide, renvoie aux questions fondamentales d'architecture protéique ; elle mettra peut-être sur la voie de maladies du cytosquelette affectant d'autres variétés cellulaires

\section{Remerciements}

Nous remercions tous les membres de l'URA 1171 du Cnrs et de l'unité 160 de l'Inserm sans lesquels le présent article n'aurait pu être écrit. Les travaux rapportés, effectués dans ces laboratoires, furent soutenus par l'université ClaudeBernard Lyon-I et l'université Paris-VI, le Centre national de la recherche scientifique (URA 1171), l'Institut national de la santé et de la recherche médicale (U 160 ; réseau Nord-Sud 489NS3), la Caisse nationale d'assurance maladie des travailleurs salariés (Contrat 8969002), le conseil de la région Rhône-Alpes.

\section{TIRÉS A PART}

J. Delaunay.

\section{Summary}

Erythrocyte skeleton and the genetic diseases of the red cell shape

The red cell skeleton is a protein assembly that laminates the inner surface of the plasma membrane. It provides the erythrocyte with the appropriate resistance and deformability. The skeletal proteins - mainly spectrin, actin and protein 4.1 -, their assembly with each other and their attachment to transmembrane proteins have been dissected. Many cDNAs have been sequenced. The exon-intron organization of the genes and their regulatory regions are under study. Red cell skeletal proteins are easily accessible representatives of ubiquitous proteins. The latter are encoded by multigenic families or obtained by alternative splicing, optional initiation of transcription or translation. Hereditary elliptocytosis and spherocytosis are genetic disorders of the red cell skeleton. They result from an amazing array of mutations. These mutations provide an approach of the structure-function relationship within the skeleton and sometimes allow to trace partially the history of the concerned populations. 